\section{Flebotomíneos de localidades rurais no noroeste do Estado do Paraná, Brasil}

\author{
Sandflies in rural localities in northwest Paraná \\ State, Brazil
}

\section{Abstract}

This study reports the results from sandfly captures at six sites in a rural area of Japurá, Paraná State, Brazil, showing the vector's presence indoors, outdoors, and in residual forest. Sandfly captures were performed with Falcão traps from 8:00 AM to 3:00 PM. At sites 195, 223, 236, and 527, three captures were performed at different times from December 2007 to January 2009. At sites 175 and 218, captures were performed from January to December 2008. 8,453 sandflies were captured, with an average of 155.40 specimens per hour. $\mathrm{Ny}$ ssomyia neivai was the predominant species. Most sandflies were captured in domestic animal shelters (75.91 per hour) and in ciliary forest (38.45 per hour) during the hottest and rainiest months, in the outdoor environment with accumulation of organic matter and inadequate disposal of household wastewater. The study emphasizes the need for regular outdoor cleaning around households and building domestic animal shelters far from residences.

Insect Vectors; Leishmaniasis; Psychodidae

\section{Wilsandrei Cella ${ }^{1}$}

Simone Cristina Castanho Sabaini de Melo ${ }^{1}$ Cristiane Machado de Oliveira Legriffon 1 Janaina Sales de Freitas 1 João Balduino Kuhl 1 Ueslei Teodoro ${ }^{1}$ Robson Marcelo Rossi 1

\section{Introdução}

As leishmanioses tegumentar e visceral estão distribuídas em 88 países, especialmente em áreas tropicais e subtropicais da Ásia, África e Américas 1 . Na América Latina, o número de casos de leishmaniose tegumentar americana (LTA) tem crescido particularmente no Brasil. Na Região Sul, ocorreram 15.268 casos de LTA, dos quais 96,5\% no Paraná, de 1980 a 2008 2, nos circuitos Paraná-Paranapanema e Ribeira ${ }^{3}$.

A ocorrência de LTA em municípios do noroeste paranaense mostra a necessidade de estudos sobre o comportamento de flebotomíneos que possam nortear os trabalhos de controle desses insetos. Assim, os objetivos desta investigação foram conhecer a fauna, os ecótopos mais frequentados e o comportamento sazonal de flebotomíneos em localidades rurais, no Município de Japurá, Estado do Paraná.

\section{Material e métodos}

O Município de Japurá localiza-se entre $23^{\circ}-24^{\circ} \mathrm{S}$ e $52^{\circ}-53^{\circ} \mathrm{O}$ (Figura 1), no noroeste do Estado do Paraná, Brasil. O clima é subtropical, com temperatura média no mês mais frio inferior a $18^{\circ} \mathrm{C}$ e a temperatura média no mês mais quente acima de $22^{\circ} \mathrm{C}$. A hidrografia faz parte da bacia do rio Ivaí e a vegetação é do tipo floresta estacional 
Figura 1

Lotes 175, 195, 218, 223, 236 e 527, onde foram feitas as coletas de flebotomíneos. Município de Japurá, Estado do Paraná, Brasil.
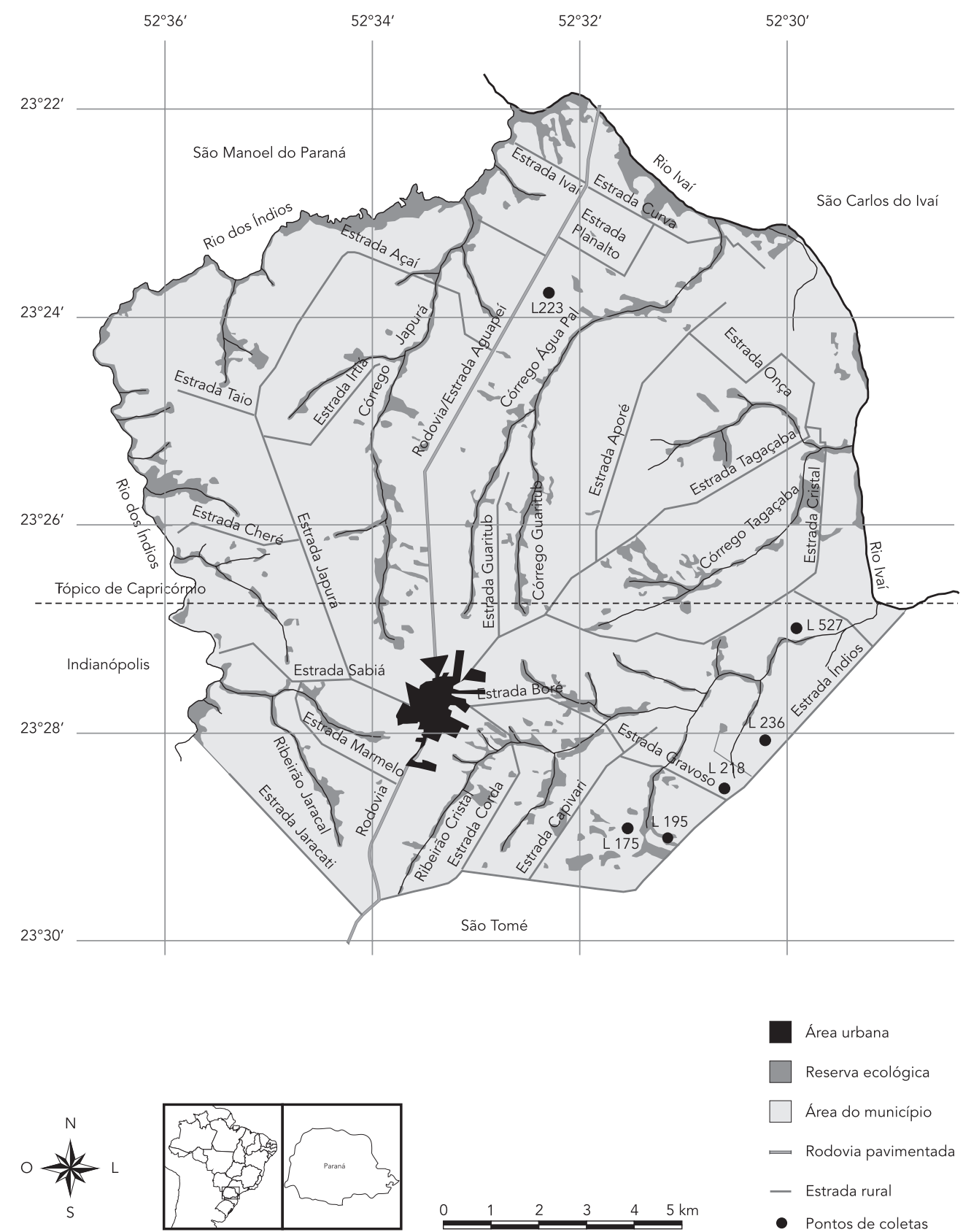

Área urbana

Reserva ecológica

Área do município

- Rodovia pavimentada

- Estrada rural

- Pontos de coletas 
semidecidual. O solo é composto por argissolo, latossolo e nitossolo vermelhos. A população estimada é de 8.247 habitantes, dos quais 7.303 vivem na zona urbana. O município tem 16.045 hectares com apenas 641,8 hectares de vegetação nativa. O cultivo de grãos ocupa a maior parte da área do município.

Os flebotomíneos foram coletados com armadilhas de Falcão 4, em módulos rurais denominados lotes (Figura 2). Os lotes 175 e 218 foram escolhidos para as coletas mensais pela facilidade de acesso e por estarem inseridos na área onde houve o maior número de casos de LTA. Nesses lotes as coletas foram feitas uma vez por mês, de janeiro a dezembro de 2008, das 20 às 3 horas, num total de 84 horas por ecótopo. Nos lotes 195, 223, 236 e 527 realizaram-se três coletas, em diferentes datas, entre dezembro de 2007 e janeiro de 2009, das 20 às 3 horas, num total de 21 horas de coletas em cada lote. O horário de coleta foi definido conforme estudos que mostram os momentos em que as densidades de flebotomíneos são mais elevadas 5,6.

As armadilhas foram instaladas em varandas de residências (3), matas ciliares (5), pomares (9), currais (3), bananais (4), pocilgas (7), galinheiros (6), pé de tamarindo (1), bambuzais (2), cafezal (1), parreira de chuchu (1), campo de futebol (1), pé de acerola (1), laranjeira (1) e ranchos (3) (Tabelas 1 e 2).

Por se tratar de um trabalho preliminar, na Tabela 1 considerou-se a soma dos flebotomíneos coletados em todos os ecótopos de cada lote. Na Tabela 2, considerou-se a soma dos flebotomíneos coletados no conjunto dos ecótopos, mesmo quando havia mais de um ecótopo do mesmo tipo (duas pocilgas, por exemplo).

Os flebotomíneos foram processados segundo Forattini et al. 7 e identificados no Departamento de Ciências Básicas da Saúde da Universidade Estadual de Maringá. A nomenclatura das espécies segue Galati 8 e as abreviaturas Marcondes 9 .

\section{Resultados}

Foram coletados 8.453 flebotomíneos das espécies Nyssomyia neivai (Pinto), Nyssomyia whitmani (Antunes \& Coutinho), Pintomyia pessoai (Coutinho \& Barretto), Migonemyia migonei (França), Pintomyia fischeri (Pinto), Brumptomyia brumpti (Larousse), Brumptomyia cunhai (Mangabeira) e Lutzomyia shannoni (Dyar) (Tabela 1). A média horária $(\mathrm{MH})$ do total de flebotomíneos capturados foi 155,40 (Tabela 1). A maioria dos flebotomíneos era fêmea, com 5.121 exemplares.
No lote 218, coletou-se a maior $\mathrm{MH}(75,16)$ de flebotomíneos, no lote 195 a segunda maior $(\mathrm{MH}=57,38)$, enquanto nos lotes 236 e 223 juntos, coletaram-se apenas 24 exemplares. Em todos os lotes, as espécies mais numerosas foram Ny. neivai e Ny. whitmani (Tabela 1).

No lote 175 as maiores $\mathrm{MH}$ de $N y$. whitmani e $N y$. neivai ocorreram em julho $(1,50$ e 1,87) e outubro $(\mathrm{MH}=0,71$ e 1,14), respectivamente. No lote 218, as maiores $\mathrm{MH}$ ocorreram em março $(0,92)$ e novembro $(0,91)$ para $N y$. whitmani e março $(39,30)$ e abril $(14,44)$ para $N y$. neivai (Figura 3).

Coletaram-se $3.272(\mathrm{MH}=65,70)$ flebotomíneos em sete pocilgas e $2.165(\mathrm{MH}=38,45) \mathrm{em}$ cinco matas ciliares. As $\mathrm{MH}$ em pomares e currais foram, respectivamente, $(12,18)$ e $(10,21)$. Em duas pocilgas do lote $195(\mathrm{MH}=35,57)$ e outra do $218(\mathrm{MH}=29,21)$, coletaram-se as maiores $\mathrm{MH}$ de flebotomíneos (Tabela 2).

\section{Discussão}

As espécies de flebotomíneos descritas em Japurá foram anteriormente assinaladas em diversos municípios do Estado do Paraná 5,10. Ny. whitmani e Ny. neivai destacam-se como espécies dominantes, pois representam 99,2\% do total de flebotomíneos coletados. Ambas as espécies são dominantes em diversas áreas endêmicas de LTA nos estados do sul do Brasil 3,10,11. A primeira espécie foi detectada com infecção natural por Leishmania no Ceará 12 e a segunda nos estados do Paraná 13 Santa Catarina 14 e Rio Grande do Sul 15 .

No lote 218, coletou-se a maioria dos flebotomíneos e foi nítido o predomínio de Ny. neivai, seguido por Ny. whitmani. Essa concentração de flebotomíneos no peridomicílio se deve à proximidade entre as pocilgas, currais, matas ciliares e a presença de uma população considerável de suínos e bovinos 3,11. Outro fato relevante nesse lote é a falta de limpeza e a presença de muita matéria orgânica e umidade, fatores que têm sido exaustivamente apontados como facilitadores da formação de criadouros de flebotomíneos no ambiente peridomiciliar e/ou nas suas proximidades $3,5,10$. Há diversos trabalhos que mostram a concentração de grande número de flebotomíneos no ambiente ocupado pelo homem e a interdependência deles com as matas remanescentes modificadas, em diversas áreas endêmicas de LTA 5,10,11.

Nos lotes 223 e 236, coletaram-se cinco e três espécies de flebotomíneos e apenas 10 e 14 exemplares, respectivamente. Em ambos os lotes, as áreas são abertas, há pouquíssimas árvo- 
Figura 2

Distribuição das armadilhas de Falcão para as coletas sazonais de flebotomíneos, nos lotes 175 e 218. Município de Japurá, Estado do Paraná, Brasil; janeiro de 2008 a dezembro de 2008.

2a) Lote 175

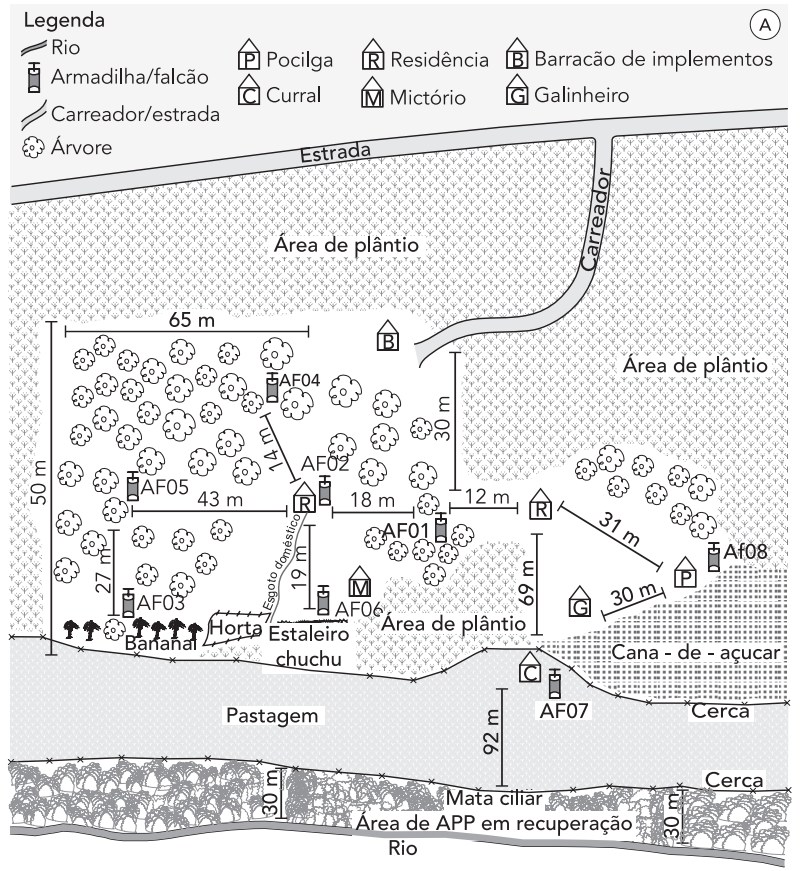

2b) Lote 218

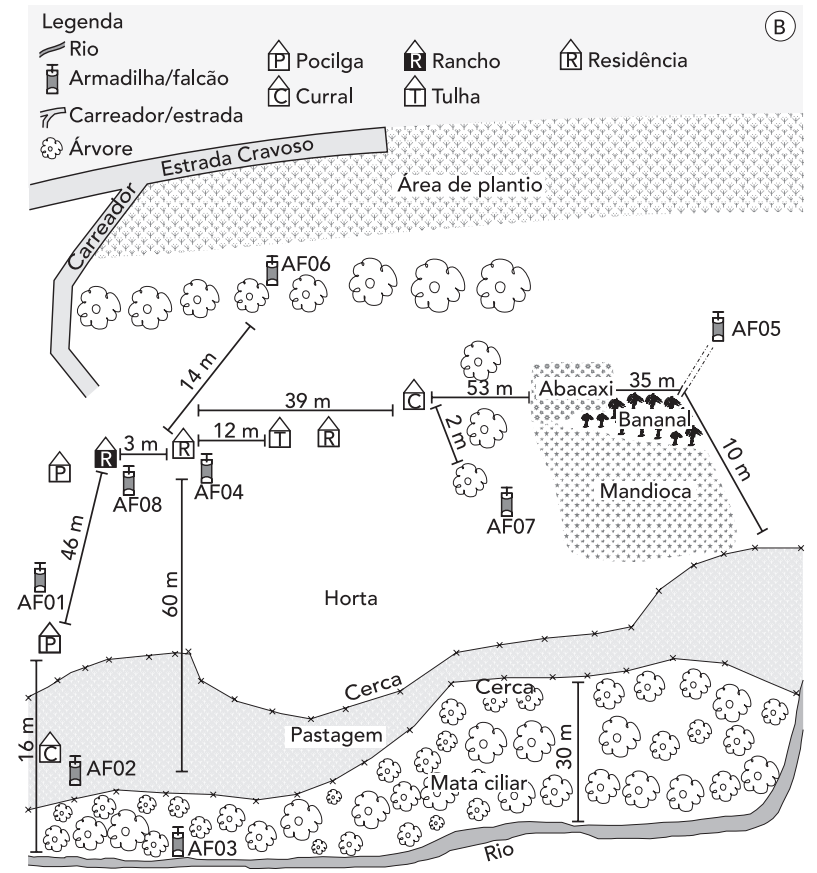


Média horária (MH) de flebotomíneos capturados em localidades rurais. Município de Japurá, Estado do Paraná, Brasil.

\begin{tabular}{|c|c|c|c|c|c|c|c|c|c|c|c|c|c|c|}
\hline \multirow[t]{2}{*}{ Espécie } & \multicolumn{2}{|c|}{ Lote 218} & \multicolumn{2}{|c|}{ Lote 175} & \multicolumn{2}{|c|}{ Lote 195} & \multicolumn{2}{|c|}{ Lote 527} & \multicolumn{2}{|c|}{ Lote 236} & \multicolumn{2}{|c|}{ Lote 223} & \multicolumn{2}{|c|}{ Total } \\
\hline & n & MH & $\mathbf{n}$ & MH & $\mathbf{n}$ & $\mathrm{MH}$ & $\mathbf{n}$ & $\mathrm{MH}$ & $\mathbf{n}$ & MH & $\mathbf{n}$ & $\mathrm{MH}$ & $\mathbf{n}$ & $\mathrm{MH}$ \\
\hline Nyssomyia neivai & 6.078 & 72,35 & 364 & 4,33 & 996 & 47,43 & 138 & 6,57 & 4 & 0,20 & 3 & 0,15 & 7.583 & 131,03 \\
\hline Nyssomyia whitmani & 216 & 2,57 & 233 & 2,78 & 188 & 8,95 & 160 & 7,62 & 9 & 0.42 & - & - & 806 & 22,34 \\
\hline Pintomyia pessoai & 10 & 0,12 & 1 & 0,01 & 4 & 0,20 & 5 & 0,23 & - & - & 4 & 0.20 & 24 & 0,76 \\
\hline Pintomyia fischeri & 5 & 0,06 & 1 & 0,01 & 5 & 0,23 & - & - & - & - & 1 & 0,05 & 12 & 0.35 \\
\hline Brumptomyia brumpti & 3 & 0,04 & - & - & - & - & 1 & 0,05 & - & - & 1 & 0,05 & 5 & 0,14 \\
\hline Brumptomyia cunhai & - & - & 1 & 0,01 & 5 & 0.23 & - & - & - & - & - & - & 6 & 0,24 \\
\hline Migonemyia migonei & 2 & 0,02 & 5 & 0,06 & 6 & 0.29 & 1 & 0,05 & 1 & 0,05 & 1 & 0,05 & 16 & 0,52 \\
\hline Psathromyia shannoni & - & - & - & - & 1 & 0,05 & - & - & - & - & - & - & 1 & 0,05 \\
\hline Total & 6.314 & 75,16 & 605 & 7,20 & 1.205 & 57,38 & 305 & 14,52 & 14 & 0,67 & 10 & 0,50 & 8.453 & 155,40 \\
\hline
\end{tabular}

Localização das armadilhas: lote 218 - pocilga (E1), curral (E2), mata ciliar (E3), varanda de residência (E4), bananal (E5), pomar-I (E6), pomar-II (E7), rancho (E8); lote 175 - pé de tamarindo (E1), varanda de residência (E2), bananal (E3), pomar-I (E4), pomar-II (E5), parreira de chuchu (E6), curral (E7), pocilga (E8); lote 195 - rancho (E1), pé de acerola (E2), pocilga-I (E3), mata ciliar-I (E4), bambuzal (E5), pocilga-II (E6), galinheiro (E7), mata ciliar-II (E8); lote 527 - laranjeira (E1), galinheiro (E2), pomar-I (E3), campo de futebol (E4), bananal (E5), pomar-II (E6), mata ciliar-I (E7), mata ciliar-II (E8); lote 236 - rancho (E1), galinheiro-I (E2), pocilga-I (E3), pocilga-II (E4), galinheiro-II (E5), galinheiro-III (E6), bambuzal (E7), pomar (E8); lote 223 - curral (E1), pocilga (E2), pomar-I (E3), cafezal (E4), pomar-II (E5), galinheiro (E6), bananal (E7), varanda de residência (E8).

Tabela 2

Média horária (MH) flebotomíneos coletados em diversos ecótopos em localidades rurais. Município de Japurá, Estado do Paraná, Brasil; dezembro de 2007 a janeiro de 2009.

\begin{tabular}{|c|c|c|c|c|c|c|c|c|c|c|c|c|c|c|}
\hline \multirow[t]{2}{*}{ Ecótopos } & \multicolumn{2}{|c|}{ Lote 218} & \multicolumn{2}{|c|}{ Lote 175} & \multicolumn{2}{|c|}{ Lote 195} & \multicolumn{2}{|c|}{ Lote 527} & \multicolumn{2}{|c|}{ Lote 236} & \multicolumn{2}{|c|}{ Lote 223} & \multicolumn{2}{|c|}{ Total } \\
\hline & $n$ & $\mathrm{MH}$ & $n$ & $\mathrm{MH}$ & $n$ & $\mathrm{MH}$ & $\mathbf{n}$ & $\mathrm{MH}$ & $\mathrm{n}$ & $\mathrm{MH}$ & $n$ & $\mathrm{MH}$ & $n$ & $\mathrm{MH}$ \\
\hline Pocilga & 2.454 & 29,21 & 69 & 0,82 & 747 & 35,57 & * & - & 2 & 0,10 & - & - & 3.272 & 65,70 \\
\hline Mata ciliar & 1.810 & 21,55 & * & - & 137 & 6,52 & 218 & 10,38 & * & - & * & - & 2.165 & 38,45 \\
\hline Pomar & 769 & 9,15 & 141 & 1,69 & * & - & 24 & 1,14 & - & - & 4 & 0,20 & 939 & 12,18 \\
\hline Curral & 670 & 7,97 & 165 & 1,96 & $\star$ & - & $\star$ & - & * & - & 6 & 0,30 & 841 & 10,21 \\
\hline Varanda de residências & 272 & 3,24 & 145 & 1,73 & * & - & * & - & * & - & - & - & 417 & 4,97 \\
\hline Bananal & 224 & 2,67 & 36 & 0,43 & * & - & 6 & 0,29 & * & - & - & - & 266 & 3,39 \\
\hline Pé de acerola & * & - & * & - & 172 & 8,20 & * & - & * & - & * & - & 172 & 8,20 \\
\hline Rancho & 115 & 1,37 & * & - & 17 & 0,81 & * & - & - & - & * & - & 132 & 2,18 \\
\hline Bambuzal & * & - & * & - & 99 & 4,71 & * & - & 3 & 0,15 & * & - & 102 & 4,85 \\
\hline Galinheiro & * & - & * & - & 33 & 1,57 & 16 & 0,76 & 9 & 0,42 & - & - & 58 & 2,75 \\
\hline Pé tamarindo & * & - & 36 & 0,43 & * & - & * & - & * & - & * & - & 36 & 0,43 \\
\hline Campo de futebol & * & - & * & - & * & - & 34 & 1,62 & * & - & * & - & 34 & 1,62 \\
\hline Parreira de chuchu & * & - & 12 & 0,14 & * & - & * & - & * & - & * & - & 12 & 0,14 \\
\hline Laranjeira & * & - & * & - & * & - & 7 & 0,33 & * & - & * & - & 7 & 0,33 \\
\hline Cafezal & * & - & * & - & * & - & $\star$ & - & * & - & - & - & - & \\
\hline Total & 6.314 & 75,16 & 605 & 7,20 & 1.205 & 57,38 & 305 & 14,52 & 14 & 0,67 & 10 & 0,50 & 8.453 & 155,40 \\
\hline
\end{tabular}

* Ecótopo inexistente.

Localização das armadilhas: lote 218 - pocilga (E1), curral (E2), mata ciliar (E3), varanda de residência (E4), bananal (E5), pomar-I (E6), pomar-II (E7), rancho (E8); lote 175 - pé de tamarindo (E1), varanda de residência (E2), bananal (E3), pomar-I (E4), pomar-II (E5), parreira de chuchu (E6), curral (E7), pocilga (E8); lote 195 - rancho (E1), pé de acerola (E2), pocilga-I (E3), mata ciliar-I (E4), bambuzal (E5), pocilga-II (E6), galinheiro (E7), mata ciliar-II (E8); lote 527 - laranjeira (E1), galinheiro (E2), pomar-I (E3), campo de futebol (E4), bananal (E5), pomar-II (E6), mata ciliar-I (E7), mata ciliar-II (E8); lote 236 - rancho (E1), galinheiro-I (E2), pocilga-I (E3), pocilga-II (E4), galinheiro-II (E5), galinheiro-III (E6), bambuzal (E7), pomar (E8); lote 223 - curral (E1), pocilga (E2), pomar-I (E3), cafezal (E4), pomar-II (E5), galinheiro (E6), bananal (E7), varanda de residência (E8). 
Sazonalidade de Nyssomyia neivai e Nyssomyia whitmani nos lotes 175 e 218. Município de Japurá, Estado do Paraná, Brasil; janeiro de 2008 a dezembro de 2008.

3a) Lote 175

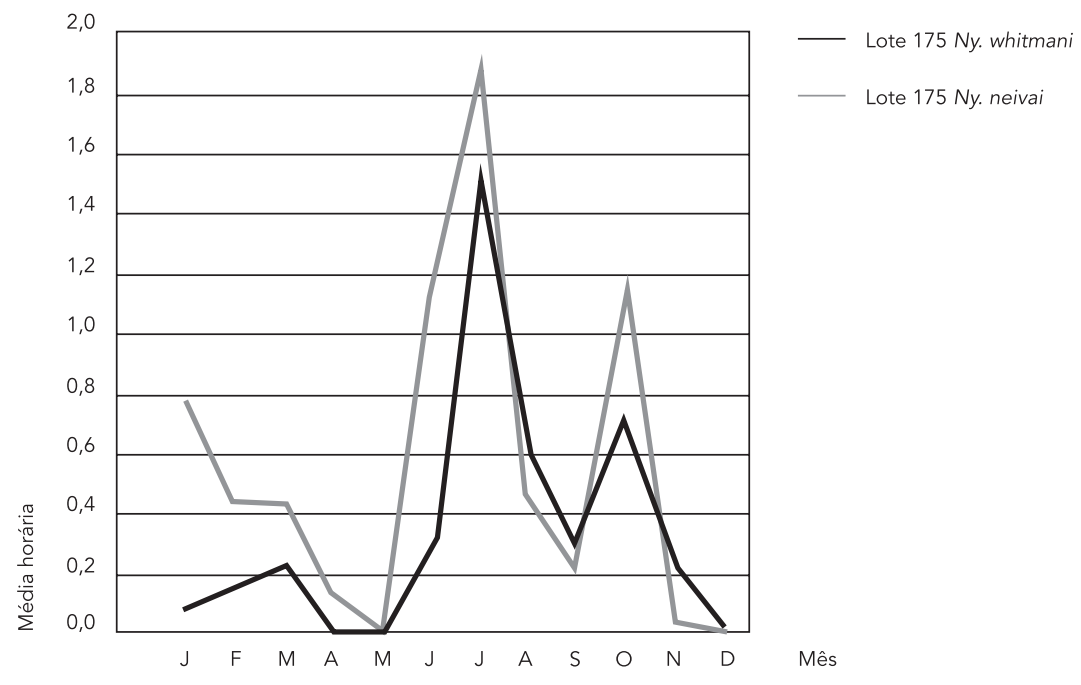

3b) Lote 218

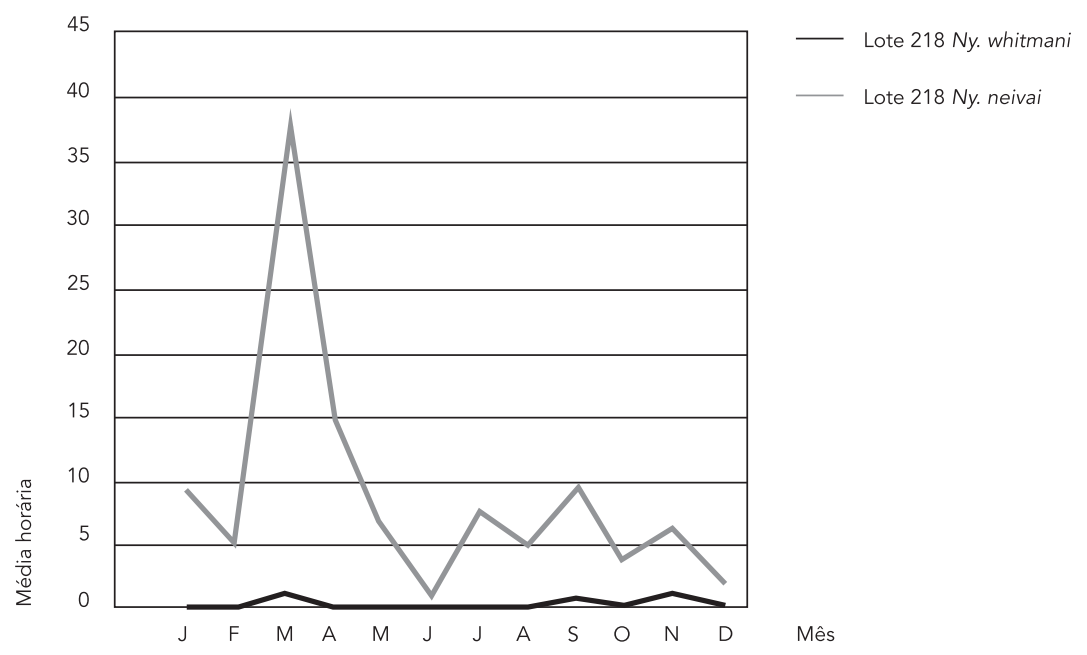

res, elevada incidência de luz solar e plantio de grãos que utilizam inseticidas para o controle de pragas. Além disso, o solo é derivado do arenito caiuá, pobre em matéria orgânica e isso podem dificultar o desenvolvimento de flebotomíneos.

No lote 218, as espécies Ny. neivai e Ny. whitmani foram coletadas em todos os meses e em maior número naqueles mais quentes e chuvo- sos, enquanto no lote 175 essas espécies foram mais numerosas nos meses mais frios e secos. No Paraná, ainda não foram estabelecidos os meses de maior transmissibilidade de Leishmania, mas acredita-se que há maior probabilidade de que eles coincidam com os meses em que há maior densidade de flebotomíneos. Contudo, não se pode descartar a existência de transmissão nos 
meses em que há menor densidade, pois quando a temperatura, principalmente a média mínima, não cai muito, mesmo nos meses mais secos, os flebotomíneos podem ser coletados em quantidades expressivas ${ }^{6}$. Embora as coletas tenham sido feitas apenas uma vez ao mês, os resultados indicam a possibilidade de que o comportamento sazonal dos flebotomíneos, no município de Japurá, não difere do que tem sido observado em outros municípios do Paraná 6,16.

\section{Resumo}

Relatam-se os resultados de coletas de flebotomíneos em seis lotes na zona rural no Município de Japurá, Estado do Paraná, Brasil, mostrando a presença de flebotomíneos no domicílio, peridomicílio e em matas residuais. As coletas de flebotomíneos foram realizadas com armadilhas de Falcão, das 20:00h às 03:00h. Nos lotes 195, 223, 236 e 527 foram feitas três coletas em diferentes períodos, de dezembro de 2007 a janeiro de 2009. Nos lotes 175 e 218, as coletas foram feitas de janeiro a dezembro de 2008. Coletaram-se 8.453 flebotomíneos, com uma média horária (MH) de 155,40 exemplares. Nyssomyia neivai foi a espécie dominante. A maioria dos flebotomíneos foi coletada em abrigos de animais domésticos $(M H=75,91)$ e nas matas ciliares $(M H=38,45)$, nos meses mais quentes e chuvosos, num ambiente peridomiciliar com acúmulo de matéria orgânicas e descarte inadequado de águas de uso doméstico, o que mostra a necessidade da limpeza regular do peridomicílio e do afastamento dos abrigos de animais domésticos das residências.

Insetos Vetores; Leishmaniose; Psychodidae
Capturaram-se oito espécies de flebotomíneos, com predomínio de Ny. neivai. Os flebotomíneos são mais numerosos em abrigos de animais domésticos, nos meses mais quentes e úmidos, num ambiente peridomiciliar com muita matéria orgânica e descarte inadequado de águas de uso doméstico. O que mostra a necessidade da limpeza regular do peridomicílio e do afastamento dos abrigos de animais domésticos das residências para evitar o contato do homem com os flebotomíneos.

\section{Colaboradores}

W. Cella colaborou na realização das coletas, identificação de flebotomíneos, organização e interpretação dos resultados e elaboração do manuscrito. S. C. C. S. Melo, C. M. O. Legriffon e J. S. Freitas contribuíram na identificação de flebotomíneos, na organização e interpretação dos resultados. J. B. Kuhl colaborou na identificação dos flebotomíneos e interpretação dos resultados. U. Teodoro orientou a realização do trabalho e participou na interpretação dos resultados e elaboração do manuscrito. R. M. Rossi participou na análise e interpretação dos resultados.

\section{Agradecimentos}

Ao Conselho Nacional de Desenvolvimento Científico e Tecnológico (CNPq, processo 410550/2006-0) pelo apoio financeiro. 


\section{Referências}

1. World Health Organization. Leishmaniasis: magnitude of the problem. http://www.who.int/leishma niasis/burden/magnitude/burden_magnitude/in dex.html (acessado em 08/Nov/2008).

2. Secretaria de Vigilância em Saúde, Ministério da Saúde. Casos de leishmaniose tegumentar americana: Brasil, Grandes Regiões e Unidades Federadas, 1990 a 2006. http://portal.saude.gov.br/ portal/arquivos/pdf/tabela_lva_casos_brasil.pdf (acessado em 30/Jan/2010).

3. Monteiro WM, Neitzke HC, Silveira TGV, Lonardoni MVC, Teodoro U, Ferreira MEMC. Polos de produção de leishmaniose tegumentar americana no norte do Estado do Paraná, Brasil. Cad Saúde Pública 2009; 25:1083-92.

4. Falcão AR. Um novo modelo de armadilha luminosa de sucção para pequenos insetos. Mem Inst Oswaldo Cruz 1981; 76:303-5.

5. Teodoro U. Características ecológicas de flebotomíneos (Diptera, Psychodidae) em habitats antrópicos, Município de Jussara, Paraná, Brasil [Tese de Doutorado]. Curitiba: Universidade Federal do Paraná; 1995.

6. Teodoro U, Alberton D, Kuhl JB, Santos ES, Santos DR, Santos AR, et al. Ecologia de Lutzomyia (Nyssomyia) whitmani em área urbana do município de Maringá, Paraná. Rev Saúde Pública 2003; 37:651-6.

7. Forattini OP, Pattoli DGB, Rabello EX, Ferreira AO. Infecção natural de flebotomíneos em foco enzoótico de leishmaniose tegumentar no Estado de São Paulo. Rev Saúde Pública 1972; 6:431-3.

8. Galati EAB. Morfologia e taxonomia. In: Rangel EF, Lainson R, organizadores. Flebotomíneos do Brasil. Rio de Janeiro: Editora Fiocruz; 2003. p. 23-51.

9. Marcondes CB. A proposal of generic and subgeneric abbreviations for phlebotomine sandflies (Diptera: Psychodidae: Phlebotominae) of the world. Entomol News 2007; 118:351-6.

10. Teodoro U, Santos DR, Santos AR, Oliveira O, Poiani LP, Silva AM, et al. Informações preliminares sobre flebotomíneos do norte do Paraná. Rev Saúde Pública 2006; 40:327-30.
11. Silva AM, Camargo NJ, Santos DR, Massafera R, Ferreira AC, Posta C, et al. Diversidade, distribuição e abundância de flebotomíneos (Diptera: Psychodidae) no Paraná. Neotrop Entomol 2008; 37:209-25.

12. Azevedo ACR, Rangel EF, Costa EM, David J, Vasconcelos AW, Lopes UG. Natural infection of $L$. (Nyssomyia) whitmani (Antunes \& Coutinho, 1939) by Leishmania of the braziliensis complex in Baturité, Ceará State, Northeastern Brazil. Mem Inst Oswaldo Cruz 1990; 85:251.

13. Oliveira DM, Reinhold-Castro KR, Bernal MVZ, Legriffon CMO, Lonardoni MVC, Teodoro U, et al. Natural infection of Nyssomyia neivai by Leishmania (Viannia) spp. In the of Paraná, southern Brazil, detected by multiplex polymerase chain reaction. Vector-Borne Zoonotic Dis 2011; 11:137-43.

14. Marcondes CB, Bittencourt IA, Stoco PH, Eger I, Grisard EC, Steindel M. Natural infection of Nyssomyia neivai (Pinto, 1926) (Diptera: Psychodidae, Phlebotominae) by Leishmania (Viannia) spp. in Brazil. Trans R Soc Trop Med Hyg 2009; 103:1093-7.

15. Pita-Pereira D, Souza GD, Zwetsch A, Alves CR, Britto C, Rangel EF. First report of Lutzomyia (Nyssomyia) neivai (Diptera: Psychodidae: Phlebotominae) naturally infected by Leishmania (Viannia) braziliensis in a periurban area of south Brazil using a multiplex polymerase chain reaction assay. Am J Trop Med Hyg 2009; 80:593-5.

16. Teodoro U, La Salvia Filho V, Lima EM, Barbosa OC, Ferreira MEMC, Silveira TGV. Flebotomíneos em áreas de transmissão de leishmaniose tegumentar na região norte do Estado do Paraná-Brasil: variação sazonal e atividade noturna. Rev Saúde Pública $1993 ; 27: 190-4$.

Recebido em 06/Ago/2010

Versão final reapresentada em 02/Ago/2011

Aprovado em 22/Ago/2011 